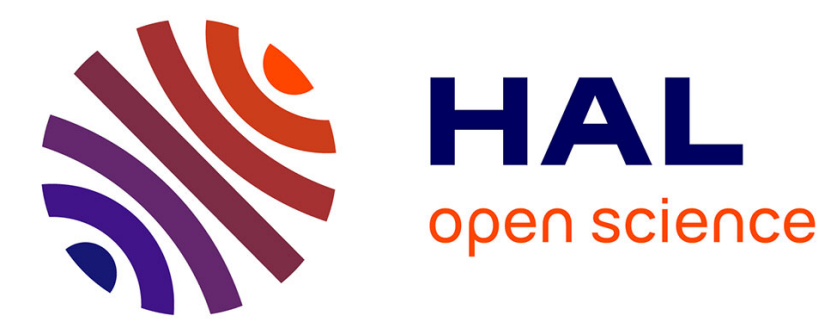

\title{
ATOMIC DATA AND SOLAR PHOTOSPHERIC SPECTROSCOPY
}

\author{
N. Grevesse
}

\section{To cite this version:}

N. Grevesse. ATOMIC DATA AND SOLAR PHOTOSPHERIC SPECTROSCOPY. Journal de Physique IV Proceedings, 1991, 01 (C1), pp.C1-181-C1-190. 10.1051/jp4:1991121 。 jpa-00249759

HAL Id: jpa-00249759 https://hal.science/jpa-00249759

Submitted on 1 Jan 1991

HAL is a multi-disciplinary open access archive for the deposit and dissemination of scientific research documents, whether they are published or not. The documents may come from teaching and research institutions in France or abroad, or from public or private research centers.
L'archive ouverte pluridisciplinaire HAL, est destinée au dépôt et à la diffusion de documents scientifiques de niveau recherche, publiés ou non, émanant des établissements d'enseignement et de recherche français ou étrangers, des laboratoires publics ou privés. 


\title{
ATOMIC DATA AND SOLAR PHOTOSPHERIC SPECTROSCOPY
}

\section{N. GREVESSE}

\author{
Institut d'Astrophysique, Université de Liège, B-4000 Cointe-Liège, \\ Belgique
}

\begin{abstract}
Résumé - Depuis la revue que nous avions faite il y a quelques années $/ 1 /$, le rôle fondamental des données atomiques et de leur précision en spectroscopie solaire n'a fait que se renforcer. Nous insistons sur l'urgent besoin pour un plus grand nombre de données atomiques très précises : longueurs d'onde, potentiels d'ionisation, fonctions de partition, structures hyperfines, probabilités de transition, sections efficaces de collisions, processus d'élargissement des rajes, facteurs de Landé, .... Nous justifions ces demandes en montrant que ces données très précises pourraient permettre une connaissance bien meilleure des conditions physiques, des processus physiques et des abondances des éléments dans les couches extérieures du soleil.
\end{abstract}

\begin{abstract}
This review is an updated version of a review we made a few years ago /1/. We insist on the role atomic data and their accuracy play in the interpretation of the solar photospheric spectrum, leading to a better knowledge of the physical conditions, physical processes and abundances of the elements in the solar outer layers. Astronomers need many more very accurate atomic wavelengths, ionization energies, partition functions, hyperfine structures, transition probabilities, cross sections, line broadening parameters, Landé g-values, ....
\end{abstract}

\section{1 - INTRODUCTION}

Solar astronomers need a large number of accurate atomic data. Stellar astronomers certainly need many more of these data. Atomic spectroscopists have actually the required experimental or theoretical techniques to fill the astronomer's needs. Why now do we ask atomic spectroscopists to spend much time refining the knowledge of the wavelengths of elements like FeI or Fell to accuracies of the order of $10^{-7}$ to $10^{-8}$ if many wavelengths are known to $10^{-6}$; why do we request transition probabilities with accuracies of $10 \%$ or better if many transition probabilities are known to within 25 to $40 \%$; why do we insist to have accurate transition probabilities for very faint lines, so faint that one hardly sees them in older solar spectra?

We shall try to answer to these questions and justify our needs for many more atomic data and for a much higher accuracy of these data by the perspectives they could open as far as our detailed description of the solar outer layers is concerned.

Solar spectra exist from the ultraviolet to the far infrared; these spectra have very high resolutions and very high signal over noise ratios. Unfortunately, they cannot be fully exploited because of the lack of the required atomic data. The sun itself is now used as a very "good laboratory source" for atomic as well as molecular spectroscopy analyses $/ 2,3,4 /$. If it is encouraging to see this close complementarity between laboratory spectroscopy and solar spectroscopy, it is also some kind of a defeat for our earth laboratories.

Our needs for accurate ionization energies and partition functions, and the role they play in solar spectroscopy analyses have been described in details in $/ 1 /$. They will not be repeated here.

We shall rather present a further request for many more data with higher accuracies in laboratory wavelengths, transition probabilities, hyperfine structures, cross sections, line broadening parameters, Landé $g$-values and illustrate the impact those data have on the solar structure and on solar physics.

\section{2 - LABORATORY WAVELENGTHS}

The top of the solar convection zone which extends from about $0.65 \mathrm{R}_{\odot}$ to the lower photospheric layers is seen through the solar granulation i.e. hot granules moving upwards together with cooler matter, the intergranular lanes, moving downwards. The observations show that lines formed at different depths in the photosphere have their wavelengths (corrected for the sun-earth Doppler shift and for the gravitational redshift) shifted to the blue relative to the laboratory wavelengths and that the line profiles are asymmetric (C-shape; see /5, 6, 7, 8, 4/. These line shifts and asymmetries can be explained if convective motions are penetrating higher layers of the photosphere, above the upper limit of the convection zone (overshooting). They result from the combined effects of the hot and cool matter weighted by the areas occupied by the hot and cool matter at each depths (see e.g. /6/). 
These apparent Doppler shifts (apparent because the observed velocities, from $100 \mathrm{~m} / \mathrm{s}$ to about $1 \mathrm{~km} / \mathrm{s}$, do not mean at all that matter is moving with the velocities derived from the line shifts and asymmetries but result from the detailed and different dependence of each tracer like FeI, Fell or CO on the temperature differences between cool and hot matter at each depth) can be transformed into real velocities of matter only through complicated hydrodynamics modelling of convection. But the observed apparent velocities are key data and severe constraints for such a modelling of the detailed structure of the dynamics of the photosphere.

Observations show that line shifts and asymmetries vary from line to line; the blue shift being the larger; the fainter the line. As faint lines are formed deeper than strong ones, it means that the convective motions are more efficient in deep layers than in the higher layers where one could naturally expect their effects to weaken and eventually disappear. A very large scatter is however observed in the apparent velocities; it is undoubtedly related to the inaccuracies of the laboratory wavelengths of the solar lines used. Furthermore, the absolute apparent line shifts are uncertain for the same reason. The modelling of the real solar structure strongly suffers from these uncertainties.

In order to be able to exploit fully the very accurate solar observations $\left(\Delta \lambda / \lambda \sim 10^{-7}\right.$ i.e. $\left.30 \mathrm{~m} / \mathrm{s}\right)$ and to measure the absolute apparent velocity shifts which are rather small (see hereabove), we need laboratory wavelengths with accuracies of the order of $\Delta \lambda / \lambda \sim 210^{-8}$ i.e. much more accurate than the ones presently available. Such data are urgently needed for Fel and FeII (see $/ 5,6,7,9 /$ ). They are also needed for EUV lines like the C IV resonance lines whose line shifts are good tracers of mass motions between the chromosphere and the corona (see /10/).

\section{LANDE g-FACTORS}

It is well known that the Zeeman splitting increases as $\lambda^{2}$ whereas the width of the lines increases as $\lambda$. The sensitivity of a line to the magnetic field will thus be proportional to $\lambda g_{L}$ ( $g_{L}$ being the Landé $g$-factor). Therefore infrared lines provide a unique opportunity to measure much weaker magnetic fields than visible lines. Zeeman patterns of visible lines often used to measure the strength and direction of the magnetic field are not resolved, making the diagnostic very difficult. Infrared lines with suitable $\mathrm{g}_{L}$ factors show, on the contrary, completely resolved Zeeman structures, allowing more precise determinations of magnetic fields.

Unfortunately, very few Landé $g$-factors are available for magnetically sensitive infrared lines. Such data are urgently needed in order to exploit fully the natural advantages of the infrared : greater precision and larger sensitivity. These data are required to make detailed models of magnetic structures like flux tubes which are observed to arise from the deep photosphere as very narrow elements (about $350 \mathrm{~km}$ ) and widen towards much higher layers where they should probably fill all the space (see e.g. $/ 11,12,4 /$ ).

\section{BROADENING PARAMETERS}

To our knowledge, little progress has been made since our previous review $/ 1 /$. The needs have on the contrary increased and we have now difficulties to explain infrared lines of FeI of very high excitation $(\sim 7.00 \mathrm{eV})$, the $4 f-5 g$ transitions recently discovered by M. Geller (private communication) and by S. Johansson /3/. Solar astronomers have also problems to explain the broadening of the so-called infrared emission lines around $12 \mu \mathrm{m}$, essentially due to Rydberg transitions in MgI, AlI, Si I and NaI (see e.g./13, 14/). The strongest of these lines actually show narrow but intense emission peaks superposed on very broad absorption troughs. Even if the formation of these lines is still a matter of debate, they are important diagnostic tools for the structures of the middle cool photosphere and of the flux tubes; they are also the most sensitive indicators of the magnetic fields in the solar spectrum (E.S. Schang, private communication; see also /4/). Because of their high excitation, a few tenths of eV below the ionization limit, both the $4 f-5 g$ FeI lines and the so-called emission lines, the strongest ones being due to $\mathrm{MgI}$, are very sensitive to the broadening mechanisms and appear much wider than other lines.

\section{HYPERFINE STRUCTURES}

The effects of hyperfine structure (HFS) on abundance analyses have been stressed in $/ 15 /$. HFS can eventually have large effects even on the abundances deduced from rather faint lines. The authors in $/ 15 /$ clearly show that we have to know, with an accuracy that is generally not available, at the same time the number of components, the component separation and the relative intensities. Neglecting the HFS or the use of an approximate pattern might lead to errors as large as a factor of 2 for a line that has an equivalent width of about $80 \mathrm{~m} \AA$ (at $5000 \AA$ ). Figure 1, where we plotted the abundance results obtained for VI lines of different strengths, with and without HFS, illustrates clearly the effects of the hyperfine structure. 


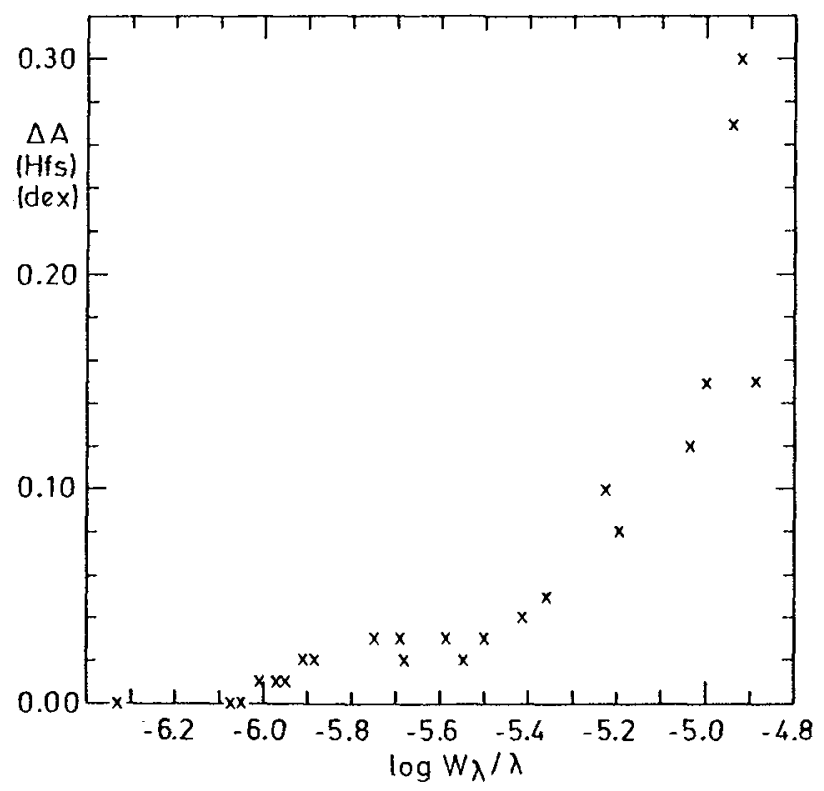

Figure 1 - Corrections to the abundance of $\mathrm{V}$, as derived from VI lines $/ 16 /$, when hyperfine structure is taken into account, as a function of the observed solar strength $\left(\log W_{\lambda} / \lambda\right)$ of the lines $[A(H F S)$ always smaller than A (without HFS)].

\section{TRANSITION PROBABILITIES}

Many groups have stressed since a long time already

(a) the importance for solar (and stellar) astronomers to have at their disposal a large number of transition probabilities, for neutral and once ionized species in the solar case, especially for faint lines and

(b) the importance of the high accuracy $(\leq 10 \%)$ actually needed for these data.

We summarized in / $1 /$ the solar applications of such high accuracy data in modelling the solar photosphere, temperature and microturbulence versus depth, in discovering the physical processus in the photosphere and in solar abundance analyses. We also gave a shopping list covering most of the important elements that is still almost up to date.

Through a few examples, we shall illustrate hereafter some of the most important impacts of highly accurate transition probabilities on the structure and physical processes of the solar photosphere.

\subsection{Solar abundances}

With the increasing accuracy of the transition probabilities, past discrepancies between meteoritic and photospheric abundances have gone away /17/. This is illustrated in figure 2 where we compare abundances recently derived for a few elements whose $g f$-values have recently been measured with high accuracy with older results obtained using exactly the same solar data but with older $g f$-values of lower accuracy available at that time. 


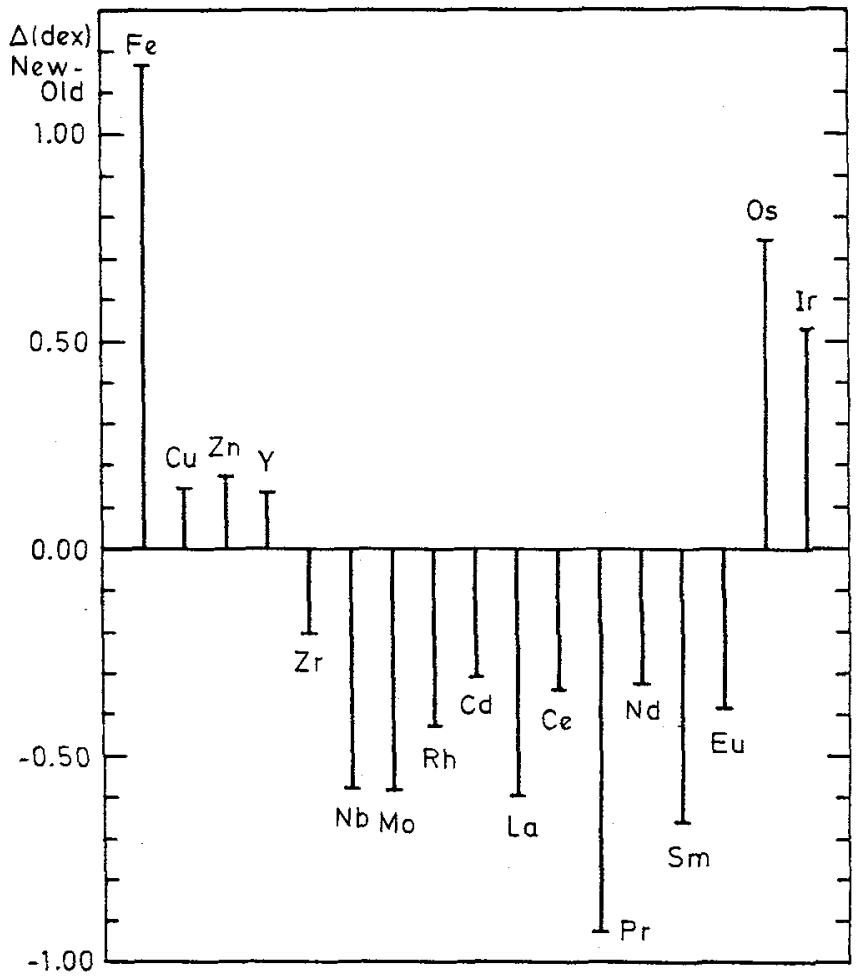

Figure 2 - Corrections to the solar abundances of a few elements that have been due to corrections to the absolute scales of the transition probabilities (new-old; the Fe correction refers to the well-known correction in 1969; other corrections are more recent).

As shown in $/ 17 /$, (see their figure 4), the agreement between meteoritic and solar abundances is now remarkably good. If we retain only 29 elements accurately known in the photosphere, having a sufficient number of good lines with accurate transition probabilities, the mean difference between the photospheric and meteoritic results is 0.000 $\pm 0.036 \mathrm{dex}$. Nevertheless there remain a few important discrepancies that have to be explained (see section 6.4).

The standard deviations of the solar abundance results (at least in the cases where a sufficient number of good quality solar lines are available) essentially mirror the quality of the transition probabilities used. This is illustrated in figures $3(\mathrm{VI})$ and $4(\mathrm{FeI})$. 


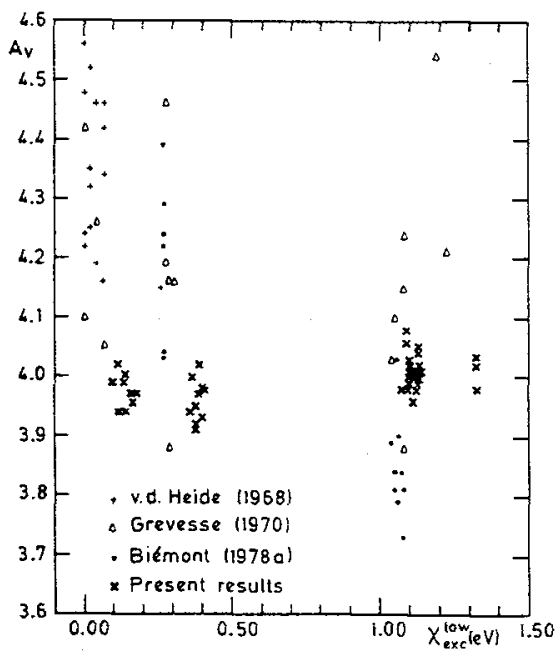

Figure 3 - Abundances of $\mathrm{V}$ as a function of the excitation energy of the lower level derived by different authors $/ 18,19,20 /$; present results $/ 16 /$. The reduction of the dispersion of the results is directly related to the increased accuracy of the transition probabilities available.

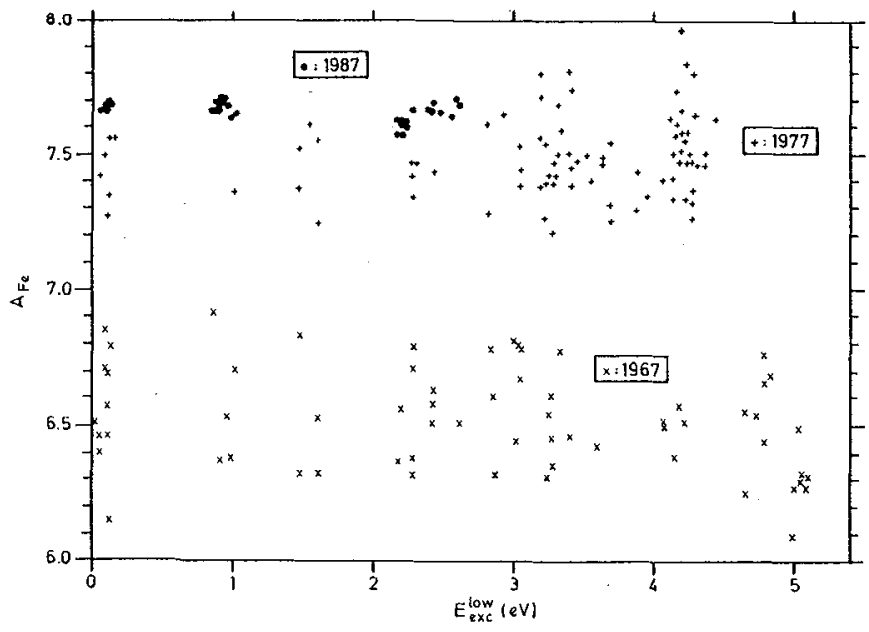

Figure 4 - Evolution of the solar abundance of iron as derived from FeI lines and of the dispersion of the results. The 1987 results are from $/ 21 /$, based on the very high accuracy Oxford $g f$-values. Earlier results are from the author, using the same solar data but $g f$-values available in 1967 and 1977 respectively.

\subsection{LTE versus non LTE}

In the past, large deviations from local thermodynamic equilibrium (LTE) have sometimes been claimed to occur in the solar photosphere, like for example in FeI. It is now certain that these pioneer results were biased because of the use of too simple atomic models and of inaccurate atomic data.

Figure 5 illustrates the present situation as derived empirically for a few elements (FeI, CrI, TiI, VI) when using very accurate transition probabilities. One clearly notices that the dispersion of the abundance results is very small and that some dependence of the abundance as a function of the excitation energy of the lower level has appeared. Such effects were totally masked when using $g f$-values of lower accuracy (see figures 3 and 4 ). The small 
variations of the abundances seen in figure 5 show that slight departures from LTE exist. The lines at $2.2 \mathrm{eV}$ in Fel that lead to a lower abundance also show the same type of behaviour in other stars $/ 24,25 /$. We can disregard possible errors in the $g f$-values of these lines. The Oxford data used in figure 5 have been confirmed recently /26/ using a different technique. These behaviours have now to be explained by careful non LTE analyses. This is a considerable step forward when compared to the situation a few years ago when a dispersion of the abundance results by a factor of about 2 (see figures 3 and 4 ) was a common place, not because of the solar data but because of the transition probabilities.

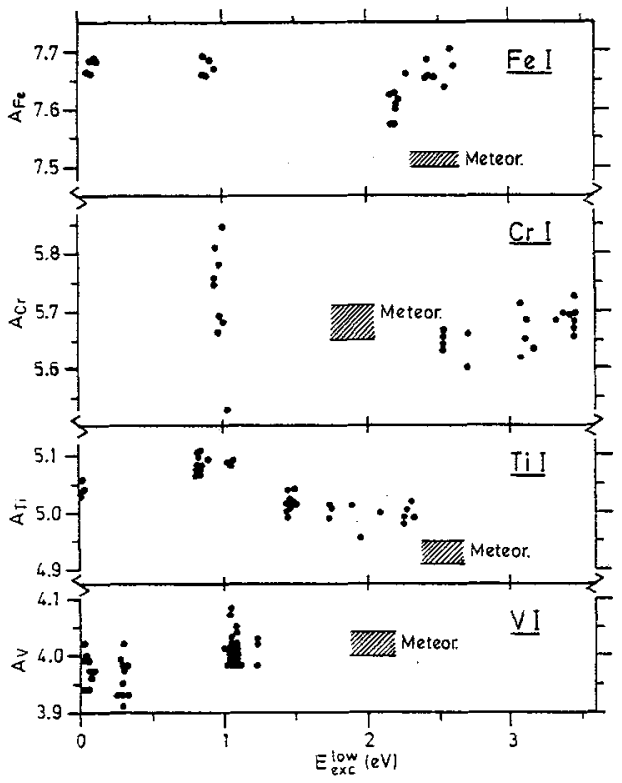

Figure 5 - Abundances as a function of the excitation energy derived from very accurate $g f$-values for low excitation lines of FeI $/ 21 /$ CrI /22/, TiI /22,23/ and VI / 16/. The variations of the abundance results with excitation energy are real and correspond to non-LTE effects (see text).

\subsection{Data required for non-LTE analyses}

Small departure from LTE in excitation seem to exist. Departures from LTE in ionization have not yet been clearly demonstrated empirically, because of the lack of a sufficient number of $g f$-values of high accuracy for two neighbouring stages of ionization (I and II) for an abundant element like Fe. If they exist, they must however be small because they are not seen in $\mathrm{V} / 16,27 /$, and $\mathrm{Y}$ and $\mathrm{ZT} / 28,29 /$ (see also /17/) for which we have accurate $g f$-values for neutral and once ionized species. Note that agreement between the abundance results derived from neutral and once ionized species does not necessarily mean that there are no non-LTE effects; it has been suggested that it might be a bias produced by the ad hoc LTE solar model used /30/. Recent solar models however seem to contradict this last suggestion $/ 4 /$.

In order to use full non-LTE formulation of the excitation-ionization equilibrium, in principle the physically correct way to treat the problem rather than making the LTE hypothesis, we need to know an extremely large number of additional physical data. In non-LTE, for analyzing one line, one needs to know a very large number of transition probabilities for a large number of transitions, cross-sections for collisional excitation and ionization by electrons, ions and hydrogen atoms, cross-sections for photoionisation, for radiative recombination, .... In LTE, on the contrary, knowledge of the $g f$-value of the line analysed, of the excitation and ionization energies and of the partition functions (mainly of the dominant stage of ionization) is sufficient to treat the problem.

These numerous data are only rarely available. They are either missing or only rough approximations are used. The question one might ask, without being able to answer to it definitely, is : is it better (in the solar case at least where non-LTE effects are small) to replace the physically uncorrect LTE approximation (which, on the other hand, seems to work so well) by the physically correct non-LTE treatment, using in this complex scheme a lot of 
atomic data of uncontrolled accuracy? The non-LTE solution might eventually be biased by the inaccuracies in the atomic data and lead to a wrong answer.

In the solar case, the FeI - FeIl equilibrium has been treated in non-tTE as carefully as possible in /30,31/; Steenbock (quoted in /31/) finds slight departures from LTE of the order of 10 to $15 \%$ in Fel lines whereas no departures are found in Fell.

Even if the departures from LTE are small, progress in our knowledge of the physical processes in the solar photosphere now requires to try and use full non-LTE treatments. In order to do this, the many data to be used (see hereabove) are needed with high accuracy.

\subsection{The case of iron and the iron group elements}

In the solar photosphere, the iron group elements are essentially once ionized. If only a few percent of FeI exist, the amount of neutrals is reduced to a few $\%$ for $\mathrm{Sc}, \mathrm{Ti}, \mathrm{V}$ and $\mathrm{Cr}$ which have much lower ionization energies than Fe. Therefore, for all these elements, the once ionized species will be rather insensitive to temperature whereas the neutrals will strongly depend on $T$. The best indicators of the abundances of these elements are obviously lines of the once ionized species but very accurate $g f$-values are generally not available. Furthermore, suitable lines, i.e. rather faint lines of good quality, are missing in the solar spectrum for some of these species.

If we are obliged to derive the abundance from lines of the neutral species of the iron group elements, we should prefer using high excitation rather than low excitation lines. They are less sensitive to $T$ than low excitation lines (a simple application of the Saha and Boltzmann laws readily shows it) and, being formed in deeper layers where the densities are higher, they should be in LTE. Although progress has been made recently, $g f$-values of high excitation lines of neutral iron group elements are still missing for many species.

Very accurate $g f$-values exist for low excitation lines of neutral iron group elements $/ 32,16,33,34,35,23,36 /$. These lines are unfortunately very sensitive to $\mathrm{T}$ and they show small departures from LTE (figure 5 ). Furthermore, some solar abundances derived from these lines disagree with the very accurate meteoritic values : $\Delta$ (phot. met.) $=0.16 \operatorname{dex}(\mathrm{Fe}), 0.06(\mathrm{Tc}),-0.14(\mathrm{Mn}) / 17 /$ (see also $/ 37 /)$.

The most important case is without any doubt this iron problem because iron has the richest spectrum in the sun, because of its high abundance and because of its meaning in stars where its abundance is often associated with the metallicity, $Z$, although it only represents less than $10 \%$ of $Z$.

We shall update the review of the iron problem made in $/ 17 /$ (see also $/ 38,39 /)$. The very accurate Oxford $g f$ values have been used by D.E. Blackwell and his collaborators $/ 21,40 /$ to derive a very accurate solar abundance of iron, $A_{F e}=7.67 \pm 0.03\left(A_{F e}=\log N_{F e} / N_{H}\right.$ in the usual scale where $\left.\log N_{H}=12.00\right)$. It is abcut $40 \%$ higher than the very accurate meteoritic value $\left(\mathrm{A}_{F e}\right.$ (met.) $\left.=7.51 \pm 0.01 / 17 /\right)$. Accurate $g f$-values for higher excitation lines of FeI have very recently been measured /26/; they will allow to see how these lines behave but it would be surprising to find so large (40\%) non-LTE effects.

Very few accurate $g f$-values exist for good FelI lines. Recent works $/ 41,42,43,44,45,38 /$ still show large disagreements between the lines in common. Solar abundances recently derived /38,39/also disagree, the result of /38/ being in agreement with the high photospheric abundance result and with the result derived from forbidden FeII lines whereas the result of /39/ agrees with the meteoritic value. The disagreement between these two results could partly be explained by the differences in $g f$-values between $/ 38 /$ and $/ 45 /$. Needless to say that very accurate $g f$-values are urgently needed for FeII; this key ion is worth special effort in view of the very important problem it could allow to solve.

\section{FAINT versus STRONG LINES}

Figure 6 shows the uncertainty of the abundance as a function of the equivalent width ( $\left.W_{\lambda}\right)$ when varying the microturbulence by $20 \%$, the uncertain damping parameter by a factor of 2 and the measured equivalent width itself by $10 \%$. It is obvious that the fainter the line (provided we can measure it with accuracy which is feasable now on the high quality solar spectra available) the smaller the uncertainty. This justifies our request for accurate $g f$-values for faint solar lines. 


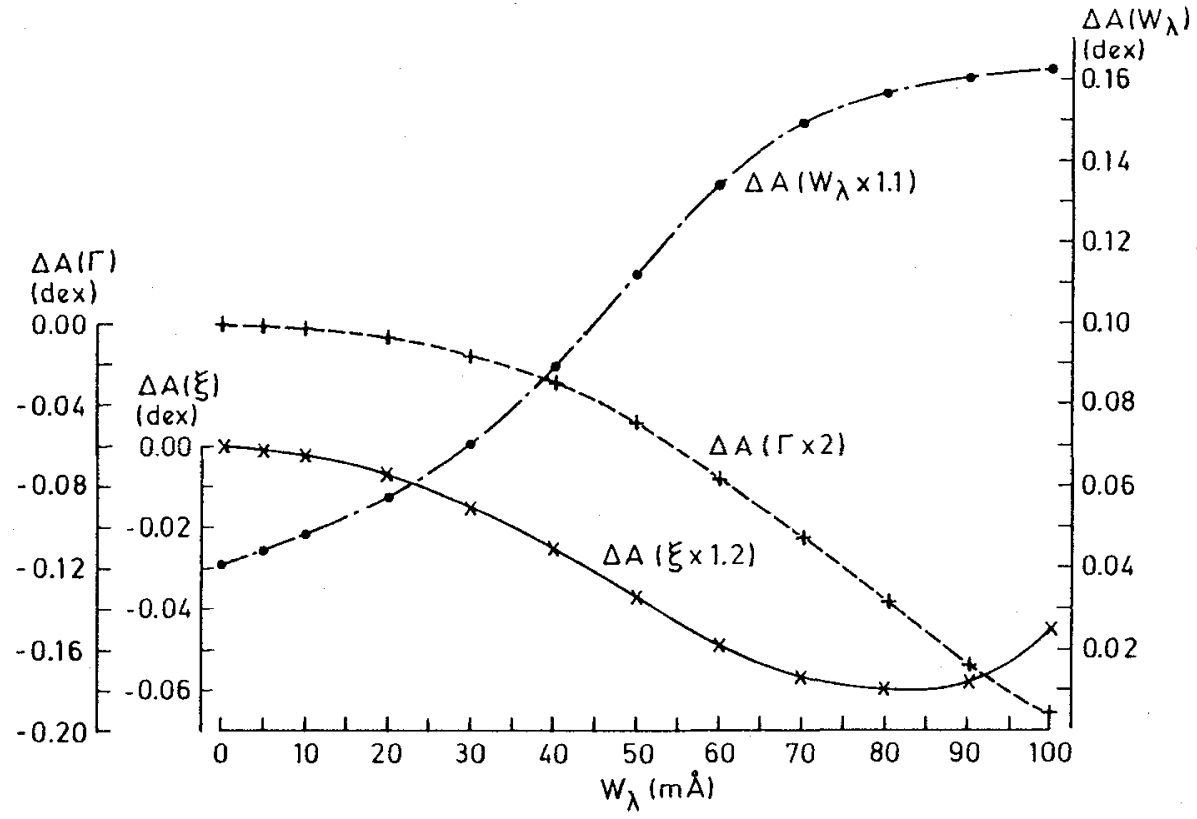

Figure 6 - Sensitivity of the abundance derived from a hypothetical FeI line $\left(5000 \AA, \mathrm{E}_{\text {exc }}=3.5 \mathrm{eV}\right)$ to uncertainties in the microturbulence, $\xi(20 \%)$, in the damping parameter, $\Gamma(\times 2)$ and in the equivalent width, $W_{\lambda}(10 \%)$, as a function of the equivalent width of the line.

\section{CONCLUSIONS}

During the last decade or so, progress has been made in our knowledge of the atomic data of solar interest, not only the number of atomic data has increased quite a lot but also the accuracy has reached the $10 \%$ level in a few cases. A very large number of data have been also computed by Kurucz /46/.

Thanks to these efforts of atomic physicists, many problems of solar physics have been solved like the general problem of the abundances of the elements in the solar photosphere which now remarkably agree with the very accurate meteoritic values (/17/; see also section 6.1 ), except in a few important cases. Thanks also to these very accurate atomic data, new problems have been discovered (sections 6.2 and 6.4); the solution to these problems requires more very precise atomic data.

Even in the cases where the situation seems to be comfortable, solar analyses show, like in the cases of NI/47/ and CI $/ 48 /$, that the atomic data and especially the best transition probabilities available are not yet of sufficient accuracy.

Solar astronomy and our knowledge of the structure and physical processes in the solar outer layers have progressed during these last decade, largely thanks to the better knowledge of atomic and molecular data. Further progress is also strongly dependent on the future improvement of our knowledge of atomic (and molecular) data. Detailed requests are to be found in $/ 1 /$ and $/ 49 /$.

A last request will concern the infrared which offers a unique opportunity to observe the solar outer layers from very deep to very high layers. New solar infrared spectra (from 2 to $16 \mu \mathrm{m}$ ) have recently been obtained from space by the ATMOS experiment /50/. These are the first observations of the true solar spectrum, i.e. not contaminated by telluric absorption, in this spectral region. They reveal the presence of a large number of very interesting atomic lines /4/ which cannot be used as tracers of the phycical conditions in the solat outer layers because of the lack of atomic data. Many of these lines have even never been observed in the laboratory. An effort should be especially made in this spectral region to fill the astronomer's needs for wavelengths, spectral classification, transition probabilities and broadening parameters (see /4/). 


\section{REFERENCES}

/1/ GREVESSE, N., Physica Scr. T 8 (1984) 49.

/2/ JOHANSSON, S. and LEARNER, R.C.M., Astrophys. J. 354 (1990) 755.

/3/ JOHANSSON, S., in The Infrared Spectral Region of Stars, ed.C. Jaschek and Y. Andrillat, Cambridge University Press (1991), in press.

/4/ GREVESSE, N. and SAUVAL, A.J., in The Infrared Spectral Region of Stars, ed. C. Jaschek and Y. Andrillat, Cambridge University Press (1991), in press.

/5/ DRAVINS, D., LINDEGREN, L. and NORDLUND, A., Astron. Astrophys. 96 (1981) 345.

/6/ DRAVINS, D. Ann. Rev. Astron. Astrophys. 20(1982) 61.

/7/ DRAVINS, D., LARSSON, B. and NORDLUND, A., Astron. Astrophys. 158 (1986) 83.

/8/ NADEAU, D., Astrophys. J., 325 (1988) 480.

/9/ DRAVINS, D., in Atomic and Molecular Data Required for Ultraviolet and Optical Astrophysics, P.L. Smith, Harvard College Observatory (1989).

10/ ORRALL, F., in Atomic and Molecular Data Required for Ultraviolet and Optical Astrophysics, P.L. Smith, Harvard College Observatory (1989).

/11/ AYRES, T.R., in Solar Photosphere : Structure, Convection, and Magnetic Fields, ed. J.O. Stenflo, Kluwer Acad. Publ., Dordrecht (1990) 167.

/12/ STENFLO, J.O., SOLANSKI, S.K. and HARVEY, J.W., Solar Phys. 173(1987) 167.

/13/ CHANG E.S., and NOYES, R.W., Astrophys. J. 275 (1983) L11.

/14/ CHANG E.S., Physica Scr. 35 (1987) 792.

/15/ BOOTH, A.J. and BLACKWELL, D.E., M.N.R.A.S. 204 (1983) 777.

/16/ Whaling, W. HANNAFORD, P., LOWE, R.M., BIEMONT, E. and GREVESSE, N. Astron. Astrophys. $153(1985) 109$.

/17/ ANDERS, E. and GREVESSE, N., Geochim. Cosmochim. Acta 53 (1989) 197.

/18/ HEIDE, von der, K., Z. Astrophys. 69 (1968) 220.

/19/ GREVESSE, N., Mém. Acad. Roy. Belgique 39 (1970) 1.

/20/ BIEMONT, E., Solar Phys. 56 (1978) 79.

/21/ BLACKWELL, D.E., BOOTH, A.J. and PETFORD, A.D., Astron. Astrophys. 132 (1984) 236.

/22/ BLACKWELL, D.E., BOOTH, A.J., MENON, S.L.R. and PETFORD, A.D., Astron. Astrophys. 180 (1987) 229.

/23/ GREVESSE, N., BLACKWELL, D.E. and PETFORD, A.D., Astron. Astrophys. 208 (1989) 157.

/24/ MAGAIN, P., Astron. Astrophys. 132 (1984) 208.

/25/ STEENBOCK, W. and HOLWEGER, H., Astron. Astrophys. 130 (1984) 319.

/26/ O'BRIAN, T.R., WICKLIFFE, M.E., LAWLER, J.E., WHALING, W. and BRAULT, J.W., preprint (1990).

/27/ BIEMONT, E., GREVESSE, N., FAIRES, L.M., MARSDEN, G., LAWLER, J.E. and WHALING W., Astron. Astrophys 209 (1989) 391.

/28/ HANNAFORD, P., LOWE, R.M., GREVESSE, N., BIEMONT, E. and WHALING, W., Astrophys. J. 261 (1982) 736.

/29/ BIEMONT, E., GREVESSE, N., HANNAFORD, P. and LOWE, R.M., Astrophys. J. 248 (1981) 867.

/30/ RUTTEN, R.J., in Physics of Formation of Fell Lines Outside LTE, ed. R. Viotti, Reidel, Dordrecht (1987).

/31/ HOLWEGER, H., in The Impact of Very High S/N Spectroscopy on Stellar Physics, eds. G. Cayrel and M. Spite, Kluwer, Dordrecht (1988) 411.

/32/ BLACKWELL, D.E., PETFORD, A.D. and SIMMONS, G.J., M.N.R.A.S. 201 (1982) 595 and ref. therein. /33/ BLACKWELL, D.E., BOOTH, H.J., MENON S.L.R. and PETFORD, A.D., M.N.R.A.S. 220 (1986) 220.

/34/ BOOTH, A.J., BLACKWELL, D.E., PETFORD, A.D. and SHALLIS, M.J., M.N.R.A.S. 208 (1984) 147.

/35/ BLACKWELL, D.E., BOOTH, A.J., MENON, S.L.R. and PETFORD, A.D., M.N.R.A.S. 220 (1986) 289 and ref. therein.

/36/ LAWLER, J.E. and DAKIN, J.T., J.O.S.A. B6 (1989) 1457.

/37/ BOOTH, A.J., Astron. Astrophys. 208 (1989) 287.

/38/ PAUlS, U., GREVESSE, N. and HUBER M.C.E., Astron. Astrophys. 231 (1990) 536.

/39/ HOLWEGER, H., HEISE, C. and KOCK, M., Astron. Astrophys. 232 (1990) 510.

/40/ BLACKWELL, D.E., BOOTH, A.J., HADDOCK, D.J., PETFORD, A.D. and LEGGETT, S.K., M.N.R.A.S. 220 (1986) 549.

/41/ MOITY, J., Astron. Astrophys. Suppl. 52 (1983) 37.

/42/ WHALING, W., Kellogg Rad. Lab., Caltech, Technical Report 84 A (1985).

/43/ KROLL, S. and KOCK, M., Astron. Astrophys. Suppl. 67 (1987) 225.

/44/ KURUCZ, R.L., Semiempirical Calculations of $g f$-values, IV : FeII, SAO Special Report 390 (1981). 
/45/ HEISE, C. and KOCK, M., Astron. Astrophys. 230 (1990) 244.

/46/ KURUCZ, R.L., Trans. I.AU. 20 B (1989) 168.

/47/ GREVESSE, N., LAMBERT, D.L., SAUVAL, A.J., van DISHOECK, E.F., FARMER, C.B. and NORTON, R.H., Astron. Astrophys. 232 (1990) 225.

148/ GREVESSE, N., LAMBERT, D.L., SAUVAL, A.J., van DISHOECK, E.F., FARMER, C.B. and NORTON, R.H., Astron. Astrophys. (1990) in press.

/49/ SMITH, P.L., Atomic and Molecular Data Required for Ultraviolet and Optical Astrophysics, Harvard College Observatory (1989).

/50/ FARMER, C.B. and NORTON, R.H., A High-Resolution Atlas of the Infrared Spectrum of the Sun and the Earth Atmosphere from Space, Vol I : the Sun, NASA Ref. Publ. 1224, NASA Scientific and Technical Information Division, Washington, D.C. 